\title{
Green Packaging Design and Finite Element Analysis of LCD Buffering Cushion
}

\author{
Shui-Gen LU1,a, Jing DONG $2, b$, Tian ZHOU ${ }^{3, c}$, Feng LIU ${ }^{4, d}$, Shuai XU \\ ${ }^{1}$ Harbin University of Commerce, Harbin 150028, China \\ ${ }^{2}$ Harbin University of Commerce, Harbin 150028, China \\ ${ }^{3}$ Harbin University of Commerce, Harbin 150028, China \\ ${ }^{4}$ Harbin University of Commerce, Harbin 150028, China \\ ${ }^{5}$ Harbin University of Commerce, Harbin 150028, China \\ alushuigen1995@163.com, bdongjing0320@163.com,c975697536@qq.com
}

Corresponding author: Jing Dong (1981), Female, Doctor, Researcher, transporting packaging, E-mail: dongjing0320@163.com

Translation author: Jia-li HUANG(1994), Female, E-mail: 1637613973@qq.com

\begin{abstract}
Keywords: buffering cushion, finite element analysis, Green design, stacking.
Abstract. Objective: To study the buffering effect of the green buffering cushion to LCD designed by the finite element analysis technology. Methods: The corrugated cardboard formed by folding was used to design the buffering cushion of the liquid crystal display. The solid model of liquid crystal display and buffering cushion was established in SolidWorks2013, and the element analysis software ABAQUS6.14 was introduced. To analysis the buffering effect of buffering cushion, this thesis carried out the simulation analysis in stacking process with finite element software. In the process of stacking simulation analysis, the stress cloud shows that there is great deformation in the connection between the liquid crystal display and the base, the stress is concentrated, and the maximum stress reaches to $0.758 \mathrm{Mpa}$, but it does not exceed the yield limit of the liquid crystal display material. The displacement cloud shows that the maximum deformation of the liquid crystal display reaches to $0.04 \mathrm{~mm}$ and the maximum deformation of the cushion is $0.22 \mathrm{~mm}$. The green buffering cushion designed by form-folding corrugated cardboard can effectively protect the liquid crystal display from damaging during the stacking process.
\end{abstract}

With the manufacturing and export growth of electronic information products in China, the packaging demand for electronic products is increasing day by day. At the same time, the cargo damage incurred in transportation process caused by improper packaging has increasingly got the attentions of enterprises and manufacturers [1,2].Generally speaking, electronic products are high value-added products, and product damage will not only increase the cost, but also make customers suspicious about the quality of the product, which damage the reputations of enterprises. Electronic products are usually fragile, easy to produce static electricity, poor tolerance in moisture and high temperature environment, so current electronic product packaging in China often uses foam polystyrene, non-woven or foam PE sheet as a cushion. However, the foaming agent Freon used in foamed polystyrene can easily damage the atmospheric ozone layer, and it is difficult to recycle and degrade after being out of use, which resulted in great environmental pollution. Due to its good characteristics like light weight, strong buffering performance, not easy to deformation, high intensity, abundant raw material sources, easy to recycle, green and environmental protection, excellent performances in damp proof and flame retardant by conducting chemical treatment and technology processing with raw materials, corrugated board is gradually replacing foam polystyrene and other materials and becoming the widely- used buffering cushion for electronic products, however, the design of corrugated cardboard cushion cushioning needs to be urgently improved [3-5].In order to better protect the product in the process of transportation and storage, enterprises usually use the method of combining actual transportation and storage, allowing damage rate and doing the product testing [6] to meet the goal of saving packaging under the premise of ensuring 
product security. But the test is time-consuming, and greatly increase the cost of packaging. So the author applied the finite element simulation to the design for the product of transportation packaging, and simulated the process of transportation and storage, analyzed parameters variation such as static displacement and stress strain [7] to obtain safe, reliable and economical packaging solutions for eliminating the risk of testing, shorting development cycle and reducing costs for enterprises.

In summary, this paper focuses on the LCD display of typical electronic products and makes innovation on green transportation packaging for corrugated cardboard which one-sheet -folding to obtain green in packing process and achieve the recycling, volume reduction, decrement and high efficiency for packaging materials.And the finite element analysis of the buffer packaging is carried out to lay the foundation for the optimal design.

\section{The Design for Liquid Crystal Display Buffering Cushion}

\subsection{The Design for Liquid Crystal Display Buffering Cushion}

Based on the consideration that the transportation packaging of the display should be buffer and shock proof in the process of transportation, the author selected the C-type corrugated cardboard featuring moderate rigidity and cushioning performance. To ensure adequate cushioning performance, the cushion is usually larger, the cost of recycling, storage and transportation are higher after being out of use, so the author adopted the corrugated cardboard sheet folding method to design the structure of the cushion, making full use of the buckle structure to make packaging materials and liner molding process green[8] and easily assembled and disassembled.The structure of liner SolidWorks is shown in Figure 1-2.

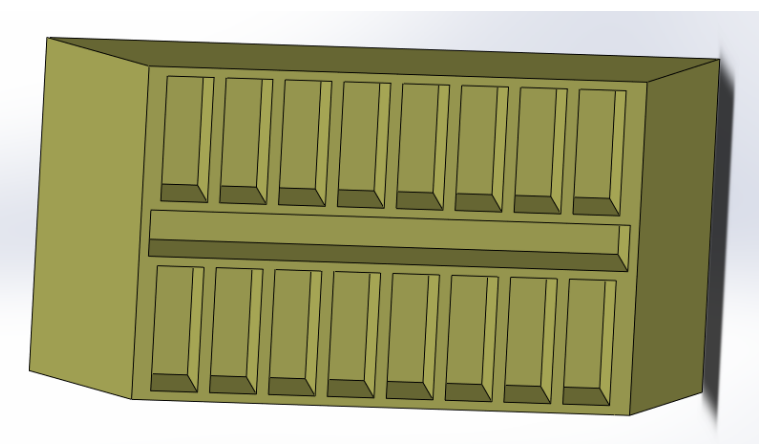

Figure.1 Top cushion model

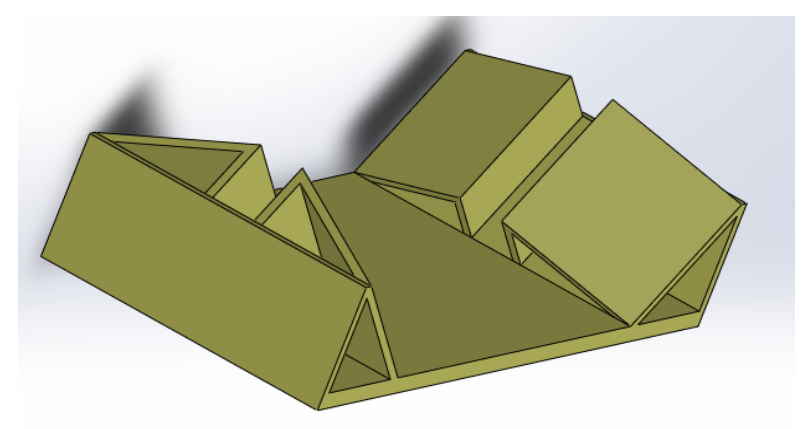

Figure.2 Bottom cushion model

\section{Liquid Crystal Display Buffer Packaging Drop Finite Element Analysis}

\subsection{The Solid Model Establishment for Liquid Crystal Display and Liner}

In order to make the finite element model closer to the actual product, the simulation process is more suitable for the actual test, the author established on the up liner, the down liner and the display frame, support frame, circuit board, external screen and other components with SolidWorks, and import finite element analysis software ABAQUS6.14, as shown in Figure 3. 


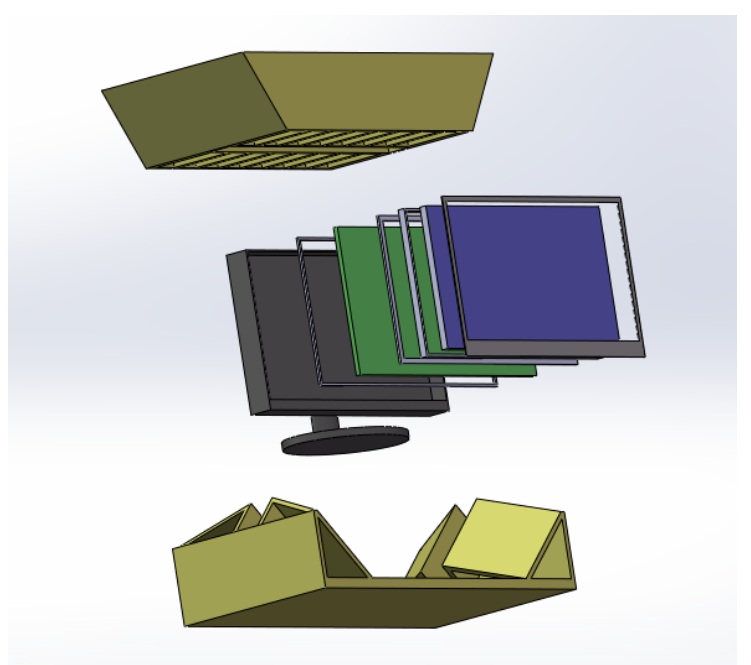

Figure.3 FEA solid model

\subsection{Material Properties Setting}

In order to make the finite element simulation analysis more reliable, the setting of material properties is very important [8]. In this paper, according to the actual characteristics of the sample material to the different components of the display, the up and down the pad of corrugated board was equivalent [9-12], as shown in Table 1 and Table 2.

Tab.1 Material Property in different parts of LCD

\begin{tabular}{llll}
\hline Name & Density $(\mathrm{kg} / \mathrm{m} 3)$ & Elastic Modulus(Pa) & Poisson's Ratio \\
\hline Support box & 7800 & 200000000000 & 0.30 \\
Support plate & 2770 & 710000000000 & 0.33 \\
Frame frame & 1070 & 2350000000 & 0.38 \\
Circuit board & 1100 & 2210000000 & 0.30 \\
Display screen & 2500 & 60000000000 & 0.25 \\
\hline
\end{tabular}

Tab.2 Material Property of corrugated board

\begin{tabular}{lllll}
\hline Density $(\mathrm{kg} / \mathrm{m} 3)$ & Elastic & Poisson's Ratio & Yield stress $(\mathrm{Pa})$ & Plastic strain \\
\hline 164.8 & Modulus $(\mathrm{Pa})$ & & & \\
& 16985000 & 0.15 & 978070 & 0 \\
& & 1078000 & 0.0041 \\
& & 1135100 & 0.01 \\
& & 1162200 & 0.0176 \\
& & 1166000 & 0.0446 \\
& & 1172800 & 0.0531 \\
& & 1192300 & 0.0607 \\
& & 1196700 & 0.0692 \\
\hline
\end{tabular}

\subsection{Simulation Test for Buffer Stacking}

\subsubsection{Calculation of Load}

In order to improve the efficiency of transport, transport packages is often to be stacked in the storage and transportation process, the package have to bear the pressure from the above goods after being stacking, and the pressure is static pressure, including deformation and creep.As shown in the formula $\mathrm{W}$, the magnitude of the static pressure is equal to the bulk weight of all the cargo in the upper layer, in that case, the bottom package receives the maximum static pressure and the static 
pressure (load) of each package depends on the weight of the unit of transport package and the quantity of its upper cargo.

It can be seen that the factor of stacking height must be taken into account when designing the hydrostatic intensity of the transport packages. Generally speaking, the stacking height of warehouse is 3 or 4 meters, in transport process, for car, the stacking height is not higher than 2.5 meters; for train, the stacking is not higher than 3 meters; for ocean ship, the warehouse is not higher than 7 meters.

Load calculation equations:

$$
\mathrm{F}=9.81 \mathrm{KM}(\mathrm{Nmax}-1)
$$

Here $\mathrm{F}$ means the carton load $(\mathrm{N})$; $\mathrm{K}$ means the load factor; $\mathrm{M}$ means the total mass of the individual carton $(\mathrm{kg})$; Nmax means the maximum stacking level.

It is known that the gross mass of the packages is $5 \mathrm{~kg}$, the maximum stacking level of the known product is 10 , and the value of $\mathrm{K}$ is 2 according to the load factor table [13]. From the equations (1) available $\mathrm{F}$ value of $822.9 \mathrm{~N}$. With F divided by the bearing surface area of carton, so the surface pressure of carton is $2943 \mathrm{~Pa}$.

\subsubsection{Simulation Test}

According to the actual conditions of product transport, the stacking height of simulation transport packages is $5 \mathrm{~m}$, the height of a single package is $0.5 \mathrm{~m}$, and the gross mass is $5 \mathrm{~kg}$.By carrying out several steps like creating parts and materials, defining cross-section characteristics, defining the assembly, setting the analysis step, defining the boundary conditions and load, dividing the grid with ABAQUS, the stacking simulation analysis of the model is carried out.Finite element grid is shown has Figure 4, stacking analysis has shown in Figure 5, the analyzing results of the stress calculation has shown in Figure 6, the analyzing results of displacement calculation has shown in Figure 7, the analyzing results of upper buffering cushion displacement has shown in Figure 8, the analyzing results of display displacement calculation has shown in Figure 9.

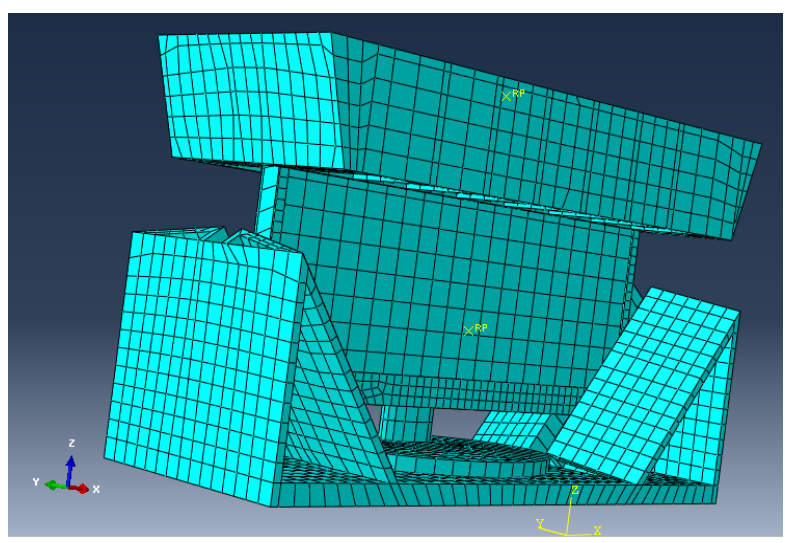

Figure.4 Finite element mesh diagram

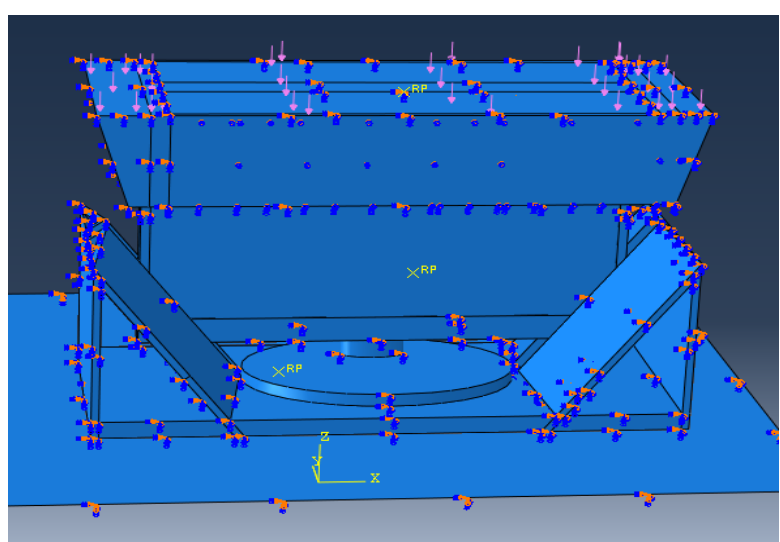

Figure.5 Stacking the applied load diagram 


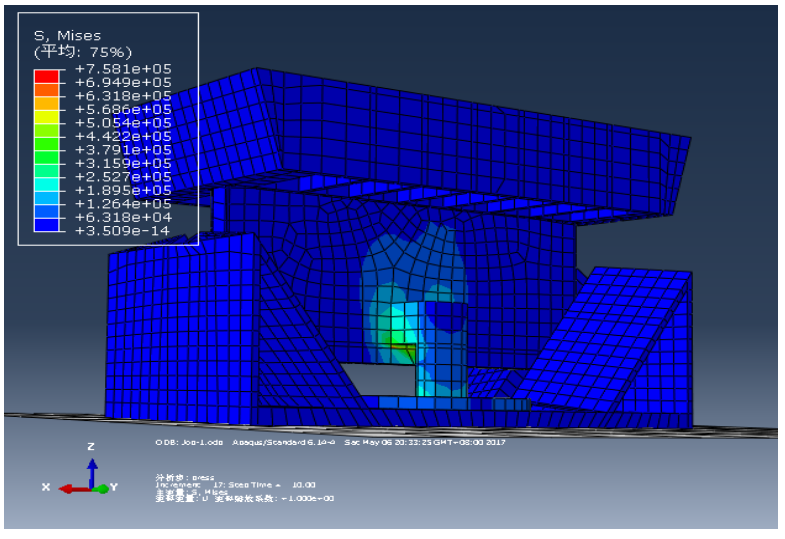

Figure.6 Stress cloud diagram

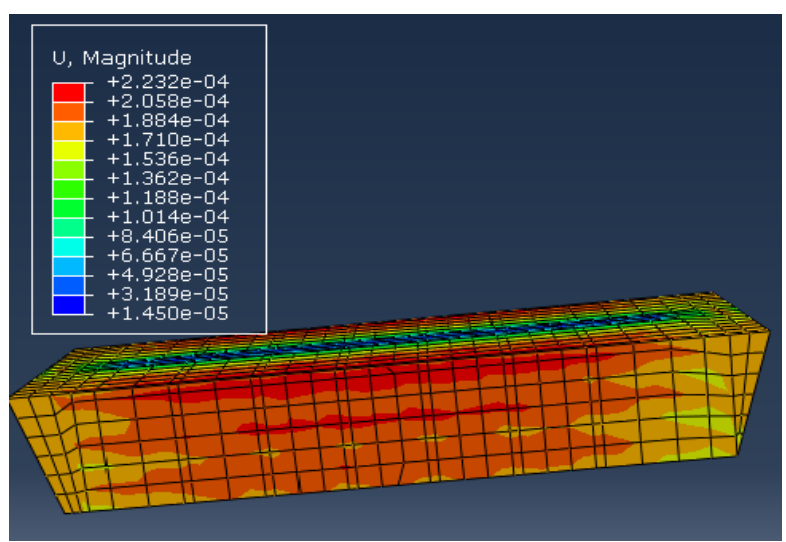

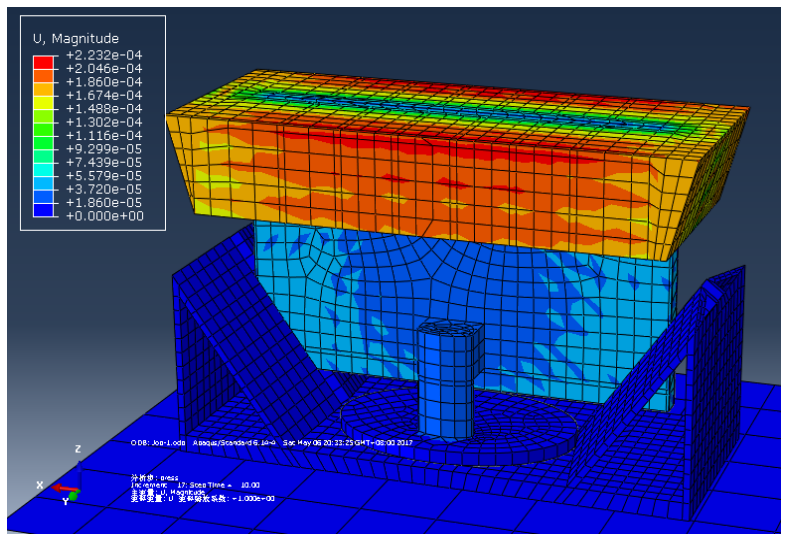

Figure.7 Displacement cloud diagram

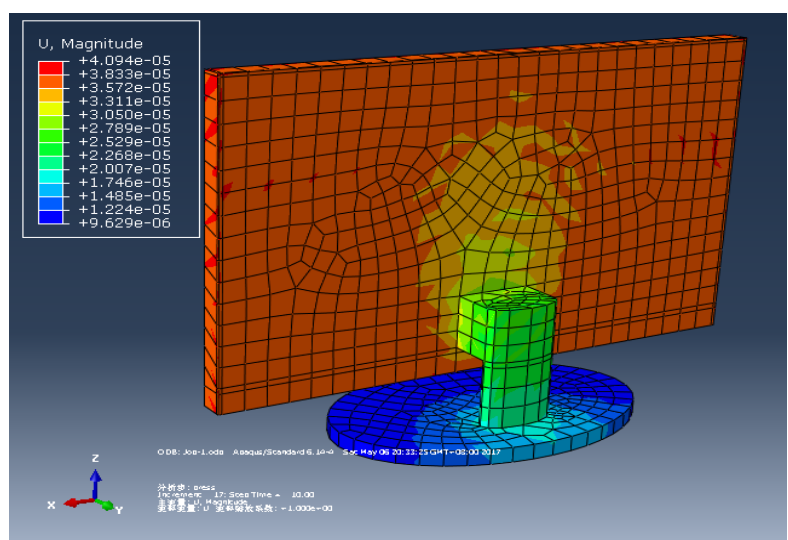

Figure.8 Top cushion Displacement cloud diagram Figure.9 Monitor Displacement cloud diagram

In the process of stacking simulation analysis, the stress cloud shows that there is great deformation in the connection between the liquid crystal display and the base, the stress is concentrated, and the maximum stress reaches to $0.758 \mathrm{Mpa}$, but it does not exceed the yield limit of the liquid crystal display material.The displacement cloud shows that the maximum deformation of the liquid crystal display reaches to $0.04 \mathrm{~mm}$ and the maximum deformation of the cushion is $0.22 \mathrm{~mm}$.It shows that the buffer pad can effectively protect the liquid crystal display from damaging during the stacking process[14].

\section{Summary}

This thesis adopted the method of corrugated cardboard formed by one sheet folding and designed the structure of the cushion, making full use of the buckle structure to make packaging materials and liner molding process green, and designed a kind of packaging liner featuring environmental friendly both in material and packaging and easy to assemble, disassemble and recycle for liquid crystal display. This thesis simulated the structures of the buffering cushion and the display with finite element software ABAQUS, established the solid models like the top and beneath liner, the external frame of the display, the support frame, the circuit board and the external screen and simulated storage of the actual conditions and carried out the stacking calculation analysis on it. According to the analysis results of stress and strain, displacement, it shows that the cushion liner is in line with requirements on the buffer packaging of display. The results of finite element simulation test are close to the experimental test, which provides a theoretical reference for the replacement of ABAQUS finite element simulation test gradually taking place with the transport packaging experiment test. 


\section{Acknowledgement}

This research was financially supported by the Innovative Entrepreneurship Project for College Students (201610240016),"Heilongjiang province ministry of education (No.12531157), Heilongjiang provincial natural science fund subject (E2016049).

\section{References}

[1] TANG Juan.Thinking of the Introduction of Green Concept in Packaging Design[J]. Packaging Engineering, 2013, 34(10): 95-98.

[2] TANG Hong-quan, ZANG Yong, LIU Yu. Features a Plurality of Green Packaging Design[J].Packaging Engineering, 2011,32(24)117-119.

[3] Joseph Sarkis, Laura M Meade, Srinivas Talluri. E-logistics and the Natural Environment[J]. Supply Chain . Management, 2004,09:303-312.

[4] PENG Guo-xun, WU Zhou-ping. The Equality of Corrugated Packaging Design[M]. Beijing:Printing Industry Press, 2007.

[5] LI Yang,CHEN Qu. Corrugated Cushioning Packaging Design of Printer Based on ANSYS[J]. Packaging Journal, 2012, (42):37-41.

[6] PENG Guo-xun. Logistics and Transport Packaging Design[M]. Beijing:Printing Industry Press, 2012.

[7] MA Guo-lu, LIU Li-xian, ZENG Guo-ying. Dropping Impact Analysis of the Packaging Structure of Changhong Television[J]. Packaging Engineering, 2008, 29(9): 6-7.

[8] DING Yu-ping, QIAN Yi. Dynamic Cushioning Property Simulation Analysis of the Overall Package Based on Ansys Workbench[J]. Packaging Engineering, 2014, 35(11): 18-22.

[9] CHE Xian-xiang. Computer Simulation on Drop Tests of Packages and Optimization of Cushion Foams Structure[D]. Jinan: Shandong University, 2013.

[10] GAO De, LU Fu-de,CHEN S J. Drop Impact Analysis of Cushioning System with an Elastic Critical Critical Component of Cantilever Beam Type[J]. Mathematical Problenms Problems in Engineering,2013,(37):1-5.

[11] QIAN Jing. Mathematical Models for Insulating Packages and Insulating Packaging Solutions[D].TN:University of Memphis,2010.

[12] YAN Shuai, LI Guang. Finite Element Analysis and Optimization Design of LCD TV Package. Packaging Engineering, 2017, 38(1): 121-127.

[13] DING Yu-ping, QIAN Yi. Dynamic Cushioning Property Simulation Analysis of the Overall Package Based on Ansys Workbench[J]. Packaging Engineering, 2014,35(11): 18 — 22.

[14] SHI Yi-ping, ZHOU Yu-rong. The Finite Element Analysis Example Explanation[J].Beijing: Mechanical Industry Press, 2006. 